\title{
Case Report: Rare Peroneocalcaneus Internus Muscle (PCI) Etiology of Tarsal Tunnel Syndrome
}

\section{Paul E Sullivan ${ }^{1 *}$, Michael P Bernstein ${ }^{2}$, Eileen L Sullivan Pharm³, Lucian M Feraru ${ }^{4}$, Jonathan S Engelhardt ${ }^{4}$ and Mitesh K Patel ${ }^{1}$}

${ }^{1}$ Department of Orthopedics, Rothman Orthopedic Institute, Thomas Jefferson University, Philadelphia, PA, USA

${ }^{2}$ Attending, Department of Podiatry, Saint Mary's General Hospital, Passaic, NJ, USA

${ }^{3}$ Assistant Professor, Saint Joseph's University, Philadelphia, PA, USA

${ }^{4}$ Resident, Department of Podiatry, Saint Mary's General Hospital, Passaic, NJ, USA

*Corresponding Author: Paul E Sullivan, Department of Orthopedics, Rothman

Orthopedic Institute, Thomas Jefferson University, Philadelphia, PA, USA.
Received: May 27, 2021

Published: June 23, 2021

(C) All rights are reserved by Paul E Sullivan., et al.

\begin{abstract}
Tarsal tunnel syndrome (TTS) can have many causes, which include direct nerve compression from cyst formation in the tarsal canal, direct trauma to the posterior tibial nerve or medial ankle impingement, fluid or tenosynovitis along the posterior tibial, flexor digitorum longus, or flexor hallucis longus tendons, varicose veins causing compression of the tibial nerve, hypertrophy of the abductor hallucis muscle belly causing compression at the level of the porta pedis, excessive pronation with arch collapse, and accessory muscles in the posterior leg. This case reports enlightens how complicated a patient presenting with heel pain can turn out to be. The article reviews tarsal tunnel syndrome and its release. It also highlights the importance of preoperative Magnetic Resonance Image (MRI) and Electromyography/Nerve Conduction Velocity (EMG/NCV) to adequately assess the cause of tarsal tunnel. The authors show the benefits of early post-op weight bearing, the ability to perform the Tarsal Tunnel Release using local anesthesia with sedation, using a high ankle tourniquet in higher-risk patients, release of the nerve proximally, especially when preoperative Valleix sign exists, as well as the use of Lyrica to decrease post-operative nerve pain.
\end{abstract}

Finally, the authors show the best stepwise approach to attack this condition and obtain the most successful surgical treatment.

Level of Evidence: Level V: Case Reports.

Keywords: Tarsal Tunnel Syndrome; Nerve Compression; Foot Surgery Techniques; Diagnostic and Therapeutic Techniques; MRI Diagnosis; Accessory Peroneocalcaneus Internus Muscle (PCI); Case Report

\section{Background}

Tarsal tunnel syndrome is a disorder frequently seen in the foot and ankle community, but its incidence is not fully understood $[1,2]$. Its etiology can be difficult to diagnose without extensive preoperative evaluation.
TTS occurs when there is a compression of the posterior tibial nerve and its associated branches, which causes pain along the medial ankle and into the plantar foot. However, some patients will report nerve pain proximal to the medial malleolus, known as a Valleix phenomenon $[3,4]$. Clinical manifestations of TTS include pa- 
resthesia, dysesthesia, and hyperesthesia radiating distally to the heel, sole, or digits [5].

These symptoms will typically present unilaterally; in approximately $10-20 \%$ of cases, patients will have bilateral pain to the tarsal tunnel [6]. EMG coupled with NCV testing has been proven to be a very effective aid in diagnosing tarsal tunnel syndrome [5,7-12]. EMG and NCV are used concurrently to help elucidate any damage or impingement on a specific nerve-in this case, the tibial nerve, as it courses through the tarsal tunnel. Additionally, MRI is an essential aid in diagnosing TTS in that it can be used to visualize space-occupying lesions such as accessory muscles, ganglion cysts, varicosities in the canal, tenosynovitis of the associated tendons in the medial ankle and soft tissue tumors, while also mapping out local inflammation and damage to the nerve itself $[4,8,10]$.

When using MRI, it is important to distinguish TTS from other causes of heel pain in adults, such as plantar fasciitis, tendinous lesions, bursal lesions, osseous lesions, peripheral nerve disorders, heel pad disorders, and infectious diseases etiologies as well as systemic disease [4,7-10]. Treatment for TTS can be conservative or surgical. Conservative options are successful in some instances and can include cortisone injection therapy at the posterior tibial nerve site, oral cortisone or anti-inflammatory agents, physical therapy, custom foot orthoses as well as Arizona-type bracing [8,10-13]. Studies have reported positive results using Lyrica as conservative treatment for patients who do not tolerate antidepressants [9].

Currently, there are no randomized controlled trials reviewing the efficacy of conservative treatment for TTS [5]. Once conservative treatment has failed to yield significant relief, surgical intervention can be considered and has been reported to have successful results.

According to Yunoki., et al. flexor retinaculum release and nerve decompression in the porta pedis have shown to be effective in relieving pain at the tarsal tunnel for 5 patients who received operative treatment. However, some of the patients reported recurring pain likely due to adhesions formed postoperatively [14]. In another study by Yu., et al. 107 patients were analyzed retrospectively and were mostly found to have good results after surgical release of the flexor retinaculum and the abductor hallucis muscle. The most significant difference in results was related to etiology, i.e. type of lesion within the tarsal tunnel [15].

\section{Case Report}

A fifty-year-old female was seen in the primary author's office complaining of pain in her left heel. Her medical history includes Type II Diabetes with early neuropathy, hypertension, and hypothyroidism. The patient is allergic to Keflex and is currently an everyday smoker. Radiographs of her foot showed no fractures, malalignment, or destructive bony process. A small calcaneal spur was noted, and the patient had concomitant heel pain consistent with a diagnosis of plantar fasciitis. EMG/NCV demonstrated prolonged distal latency in the left lateral plantar nerve, suggestive of left tarsal tunnel syndrome, signs of left L4-5 radiculopathy, and right $\mathrm{S} 1$ radiculopathy. The patient was sent for a preoperative MRI to assess the etiology of TTS further. The MRI was read as moderate focal tendinosis of the tibialis posterior tendon proximal to its attachment to a nonedematous os naviculare without tendon tearing. A peroneocalcaneus internus muscle and tendon were identified, described as an anatomic variant with one percent prevalence and found to be seventy-five percent bilateral. PCI may indirectly compress the tarsal tunnel predisposing to tarsal tunnel syndrome. Prior to surgical intervention The American Orthopedic Foot and Ankle Score (AOFAS) score was 12/100.

Conservative treatment was attempted, including orthoses, injections, physical therapy, ice, and oral medications. At this point, conservative treatment was exhausted, and the patient desired surgical treatment. Risks of procedure were discussed, including scar tissue formation, persistent pain and numbness to foot and ankle, difficulty in shoe gear, and the possibility of additional surgery in the future.

\section{Operative technique}

The patient was placed on the operating table in a lateral decubitus position. Local anesthesia was administered using a one-toone mix of Lidocaine and Marcaine plain. The patient's lower extremity was then scrubbed, prepped, and draped in the usual aseptic technique. An esmarch was then used to exsanguinate the leg, and the mid-calf tourniquet was inflated to $300 \mathrm{mmHg}$.

A linear longitudinal incision was made at the posterolateral aspect of the distal leg, approximately $2 \mathrm{~cm}$ lateral to the Achilles tendon. Blunt dissection was then used to identify and reflect the peroneal tendons laterally. The Achilles was identified and retrac- 
ted medially. Deep to that, the peroneocalcaneus muscle belly was identified and released from the surrounding muscular tissues proximally and distally as it turned into a tendinous structure. The entire variant muscle was then removed in total.

A curvilinear incision was then made over the tarsal tunnel, which measured $4 \mathrm{~cm}$ in length. The retinaculum was resected, and the tibial nerve was identified and split into its main branches. The medial, lateral plantar nerves and the inferior medial calcaneal nerve were separated and retracted using vessel loops. Multiple venae comitantes were identified, crossing over the tibial nerve strangulating it. These were resected, bovied and tied. The flexor retinaculum was released proximally and distally to the level of the porta pedis. The origin of the Abductor hallucis was partially resected and finger dissection was utilized to ensure adequate release.

The patient was also treated for plantar fasciitis by performing an endoscopic plantar fasciotomy.

Both incisions were closed with 3.0 vicryl subcutaneously. Note flexor retinaculum should not be approximated after release. The skin was closed with skin stables due to tourniquet time, and both incisions were dressed with bacitracin and compressive dry sterile dressing (DSD). The patient was given a $75 \mathrm{mg}$ Lyrica immediately in the recovery room and discharged full weight-bearing. Post-op diclofenac $75 \mathrm{mg}$ twice a day (BID) for 10 days, Lyrica $75 \mathrm{mg} 1$ - 2 a day for burning/shooting pain PRN and Percocet 5/325 only 8 tabs dispensed as needed for severe pain.

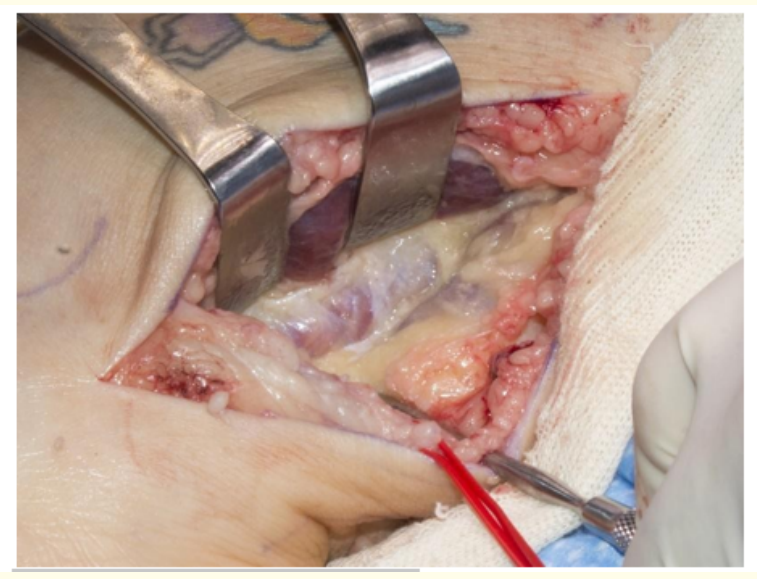

Figure 1: Identification of the PCI.

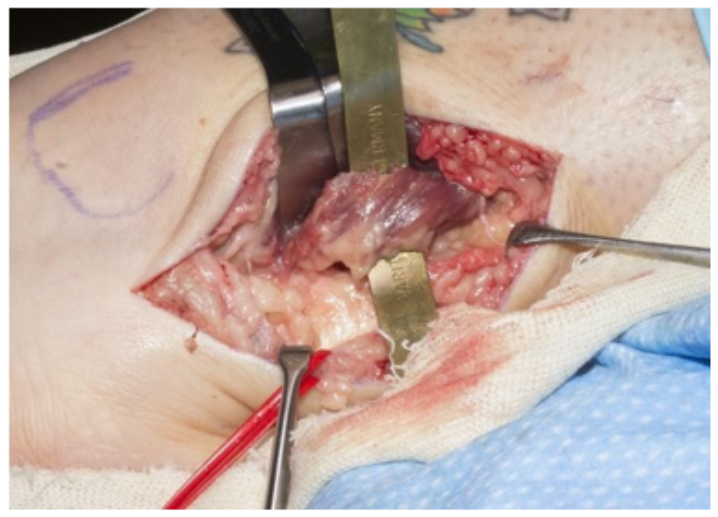

Figure 2: Isolation of the PCI.

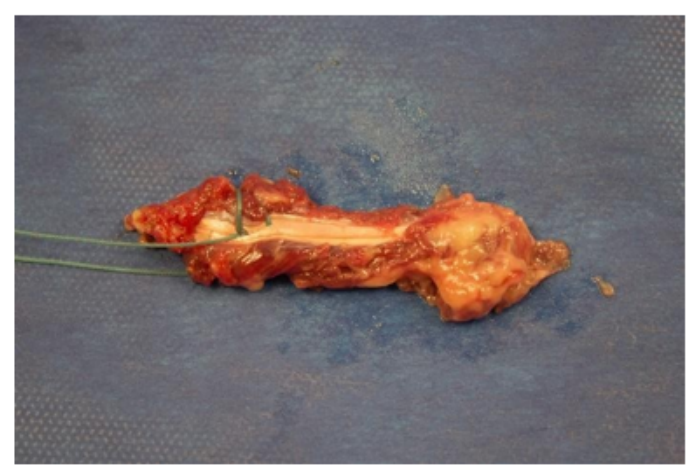

Figure 3: Removal of PCI.

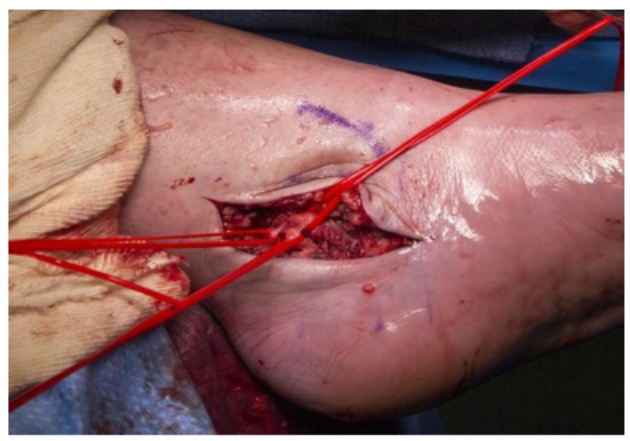

Figure 4: Dissection of the tarsal tunnel with looped posterior tibial nerve. 


\section{Discussion}

Tarsal tunnel syndrome can be effectively treated with surgical intervention, as indicated in multiple studies. According to Radin., et al. over $90 \%$ of patients with tarsal tunnel syndrome had symptoms resolved after the release of the flexor retinaculum [16]. Similarly, a study by Reade., et al. showed successful outcomes after surgical release of the retinaculum, citing improvement in $85 \%$ to $90 \%$ of cases [17]. Although the surgical release of the flexor retinaculum appears to be successful, finding the etiology of the nerve compression is vital to a successful outcome. Once a diagnosis has been reached through clinical observation, we recommend ancillary testing with EMG and nerve conduction and MRI to evaluate adjacent structures. Although not common, tarsal tunnel syndrome can be seen in the absence of a positive EMG and nerve conduction study.

This will usually occur when the nerve compression is due to varicosities or dilated veins in the tarsal tunnel canal. In these instances, patients' complaints will be noted later on in the day as the veins tend to swell. They will usually not have symptoms early in the day and during ambulation. In these situations, clinical diagnosis cannot be solely corroborated with a positive EMG and nerve conduction test. A preoperative MRI will assist in evaluating the tarsal canal and surrounding structures to localize any additional pathology to be addressed at the time of the tarsal tunnel release [4,7-10]. Our authors have seen chronic tenosynovitis of the adjacent tendons, ganglion cyst formation of the flexors or posterior tibial tendons that may be buried deep to the skin, an increase in venae comitantes or varicosities in the tarsal canal, as well as accessory muscles in the posterior ankle and leg causing significant compression to the tarsal tunnel region.

Mechanical factors must also be recognized. Such as excessive pronation causing a hypertrophic abductor hallucis muscle belly at the origin with a narrowing of the porta pedis; however, it may be challenging to interpret and discern from other causes of heel pain such as plantar fasciitis. Preoperatively, we recommend compressing the tarsal canal and marking the proximal portion of the canal that elicits pain on palpation, especially when the preoperative valleix sign is noted. This should be the proximal start of the surgeon's incision, and it should extend plantarly to the level of the porta pedis. This will make sure the nerve is adequately released proximally. Once dissected to the level of the tarsal canal, any ve- nous structures compressing the nerve should be cauterized and hand-tied as needed. The nerve will usually be found just underneath the venous and arterial vessels. Once the nerve is identified, it should be traced distally, identifying all three branches (medial, lateral, and inferior medial calcaneal branch). These branches should be separated and tagged using vessel loops while further dissection is performed to ensure each branch is adequately released and completely free of any additional compression. If there was a positive Valleix sign, the retinaculum should be resected proximally, and finger dissection should be used to free up the proximal canal. Additionally, the porta pedis should be freed up, and the abductor hallucis muscle should be examined for tightness and hypertrophy. Care should be taken when debriding the abductor hallucis due to the increased propensity of intramuscular bleeding. Once the canal has been freed up proximally and distally, and any underlying cyst or hypertrophic muscle belly has been addressed, closure of the surgical site is performed, leaving the retinaculum resected to avoid recurrent compression of the nerve.

A tourniquet is typically employed and is used at the calf level to aid in exsanguination of venous blood in the calf muscles and allow for a bloodless field that aids in visualization and reduces blood loss. Post-operative cast immobilization is not necessary for a successful outcome. In the author's experience, compression dressing, early range of motion, and weight-bearing will help prevent scarring in the area. A single crutch or cane may be used for added balance if needed. A one-time order of $75 \mathrm{mg}$ Lyrica is recommended in the post-operative period, which will avoid the need for excessive post-op narcotic use. An additional prescription for Lyrica $75 \mathrm{mg}$ once or twice a day for the first 7 - 10 days may be used to aid with any post-operative nerve pain.

\section{Conclusion}

Tarsal tunnel syndrome is a complex disorder that requires thorough investigation to elucidate its etiology. The diligent use of ancillary studies is essential to ensure the best procedure is performed to result in the possible patient outcomes. It is important to have thorough preoperative planning, including EMG/NCV followed by a diagnostic MRI prior to surgery. As previously discussed, positive EMG/NCV is not definitive for TTS as the clinical exam can reveal TTS in the absence of a positive EMG and NCV study. Additionally, the surgeon should keep in mind that these procedures can also be performed under local anesthesia with sedation. The 
placement of a high ankle tourniquet, especially when the patient is at increased risk for general or spinal anesthesia. Intraoperatively adequate release of the nerve must be completed with all three nerve branches identified completely freed from any tissue and isolated with vessel loops. It is important to preoperatively mark the highest point where pain is elicited on the posterior tibial nerve to allow for proper height of incision placement. This will identify the approximate area where the surgeon should release the flexor retinaculum, which will relieve any remaining compression or strangulation of the nerve. This should also be performed distally at the porta pedis to ensure adequate release. Additional diagnoses must be pursued such as accessory muscles in the posterior compartment of the leg (as reviewed in this case with an accessory peroneocalcaneus internus muscle), cyst formation, fluid within the Flexor or Posterior Tibial tendons, or significant strangulation of the nerve as seen with vena comitantes or varicosities of the greater saphenous vein. Early post-operative weight bearing along with range of motion will help prevent scarring in the post-operative period. The use of Lyrica 50 or $75 \mathrm{mg}$ immediately postoperatively and during the recovery period is recommended to ease pain and allow for quicker recuperation.

\section{Financial Disclosures}

No funding or support was received for the collection, analysis, interpretation of data or preparation of the article.

\section{Conflict of Interest}

The authors report no conflict of interest regarding the data presented in this article.

\section{Bibliography}

1. TA Patel., et al. "Usefulness of electrodiagnostic techniques in the evaluation of suspected tarsal tunnel syndrome: an evidence-based review". Muscle and Nerve 32.2 (2005): 23640.

2. L Llanos., et al. "Clinical symptoms and treatment of the foot and ankle nerve entrapment syndromes". Foot and Ankle Surgery 5.4 (1999): 211218.

3. H Kopell and W Thompson. "[Peripheral entrapment neuropathies of the lower extremity]". New England Journal of Medicine 262 (1960): 5660.

4. SK Singh., et al. "The surgical treatment of tarsal tunnel syndrome”. The Foot 15.4 (2005): 212216.
5. SC McSweeney and M Cichero. "Tarsal Tunnel Syndrome, a narrative review of the literature". Journal of Foot and Ankle Research 8.S2 (2015).

6. J Jerosch., et al. "Results of surgical treatment of tarsal tunnel syndrome". Foot and Ankle Surgery 12.4 (2006): 205208.

7. P Reichert., et al. "Results of surgical treatment of tarsal tunnel syndrome". Foot and Ankle Surgery 21.1 (2014): 269.

8. JS Gould. "Tarsal tunnel syndrome". Foot and Ankle Clinics 16.2 (2011): 27586.

9. L Schon and D Baxter. "Neuropathies of the foot and ankle in athletes". Clinics in Sports Medicine 9.2 (1990): 489509.

10. M Kinoshita., et al. "The dorsiflexion-eversion test for diagnosis of tarsal tunnel syndrome". Journal of Bone and Joint Surgery 83.12 (2001): 18359.

11. Reade D., et al. "Tarsal tunnel syndrome". Clinics in Podiatric Medicine and Surgery 18.3 (2001): 395408.

12. T Aldridge. "Diagnosing heel pain in adults". American Family Physician 70.2 (2004): 3328.

13. E Radin. "Tarsal tunnel syndrome". Clinical Orthopaedics and Related Research 181 (1983): 16770.

14. X Yu., et al. "Surgical efficacy analysis of tarsal tunnel syndrome: a retrospective study of 107 patients". Cell and Tissue Banking 22.1 (2020): 115122.

15. M Yunoki. "Analysis of Surgical Cases of Tarsal Tunnel Syndrome in Our Department: Case Series and Literature Review". Asian Journal of Neurosurgery 15.1 (2020): 5964.

16. WH Gondring., et al. "Tarsal tunnel syndrome: assessment of treatment outcome with an anatomic pain intensity scale". Foot and Ankle Surgery 15.3 (2008): 1338.

17. W Cimino. "Tarsal tunnel syndrome: review of the literature". Foot and Ankle International 11.1 (1990): 4752.

Volume 4 Issue 7 July 2021

(c) All rights are reserved by Paul E Sullivan., et al. 